Rechtsmedizin $2019 \cdot 29: 159$

https://doi.org/10.1007/s00194-019-0307-5

Online publiziert: 18. Februar 2019

(c) Springer Medizin Verlag $\mathrm{GmbH}$, ein Teil von Springer Nature 2019

\author{
A. Büttner $\cdot$ R. Dettmeyer ${ }^{2}$ \\ 'Institut für Rechtsmedizin, Universitätsmedizin Rostock, Rostock, Deutschland \\ ${ }^{2}$ Institut für Rechtsmedizin, Universitätsklinikum Gießen \& Marburg GmbH, Justus-Liebig Universität \\ Gießen, Gießen, Deutschland
}

\title{
Histopathologie des Drogentodes
}

elle Literatur und Fachbücher. Sieht man von den chronischen, häufig über viele Jahre klinisch symptomlosen Organschäden ab, so dürften insbesondere drogenassoziierte Organinfarkte und Infektionen auch für Ärztinnen und Ärzte von Interesse sein, die Drogenpatienten behandeln und die besonderen Risiken kennen sollten: das Risiko einer HIV-Infektion, das Risiko einer Hepatitis B/C, infektiöse Endokarditiden, Peritonitiden bei inguinalen Injektionen sowie Pannikulitiden und Myositiden; speziell beim Konsum von Kokain muss an das erhöhte Risiko von Organinfarkten gedacht werden, beim Konsum von z. B. „Ecstasy“ im Einzelfall zusätzlich an eine maligne Hyperthermie mit Rhabdomyolyse und akutem Nierenversagen.

Wichtig ist die rechtsmedizinische Erkenntnis, dass ein Risiko für auch tödliche organische Erkrankungen bzw. Reaktionen nicht zwingend korrelieren muss mit der gemessenen Blutkonzentration einer oder mehrerer Drogen, sondern dass im Einzelfall die Konsumdauer, organische Vorschäden, synergistische Effekte von Drogen und immunologische Prozesse bis zum allergisch-anaphylaktischen Schock bedacht werden müssen. Rechtsmedizinische Kasuistiken können hier einen wichtigen Beitrag zur weiteren Aufklärung leisten.

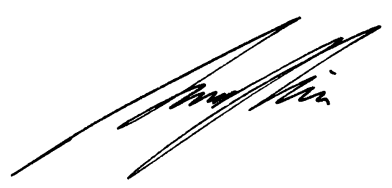

Prof. Dr. A. Büttner
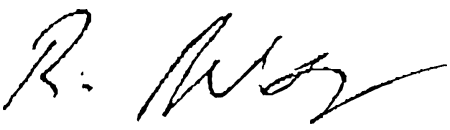

Prof. Dr. Dr. R. Dettmeyer

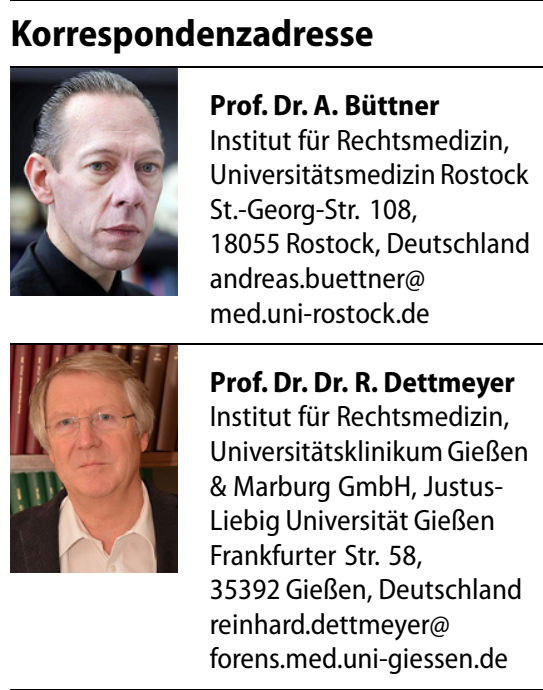

Interessenkonflikt. A. Büttner und R. Dettmeyer geben an, dass kein Interessenkonflikt besteht.

Eine orientierende Übersicht zu den wesentlichen histopathologischen Veränderungen sollen die Beiträge im vorliegenden Themenheft geben, zu allen Themen gibt es inzwischen weiterführende spezi- 